\title{
LATTICE-PARTICLE MICROSTRUCTURAL MODEL FOR ION DIFFUSION IN GRAPHITE ELECTRODE BATTERIES
}

\section{JORGE MARIN-MONTIN ${ }^{1}$, CARLOS FRESNEDA-PORTILLO ${ }^{2}$ AND FRANCISCO MONTERO-CHACÓN ${ }^{1}$}

${ }^{1}$ Universidad Loyola Andalucía, Departamento de Ingeniería, Avda. de las Universidades, s/n, 41704 Dos Hermanas (Spain), \{jjmarin@uloyola.es, fpmontero@uloyola.es\},www.uloyola.es

${ }^{2}$ Universidad Loyola Andalucía, Departamento de Métodos Cuantitativos, Avenida de las Universidades, s/n, 41704 Dos Hermanas (Spain), cfresneda@uloyola.es, www.uloyola.es

Key words: particle methods, ion diffusion, graphite electrodes, batteries

\begin{abstract}
In this work, we propose a lattice-particle approach to study ionic diffusion across graphite electrodes. In our approach, we generate virtual representative volume elements (RVE) of the electrode material based on its composition, i.e., active particles, carbon additives, and binder. Porosity is also accounted for as an input parameter. To account for the evolution of the ionic concentration, Fickean diffusion is considered. This problem is solved within a network of one-dimensional elements, which is constructed upon the particles of the RVE, yielding a three-dimensional lattice. We use the centraldifference time-integration scheme to solve the transient problem within the framework of the finite element method for the spatial discretization. One of the main advantages of our approach is that we are able to reduce the number of degrees of freedom and thus the computational cost in comparison to the conventional continuum-based finite element simulations. For the transport simulations, we consider $\mathrm{Li}$ ions, although our approach can be also applied to other type of species, such as $\mathrm{PF}_{6}^{-}$anions in the case of DIGB with $\mathrm{LiPF}_{6}$ electrolyte, for instance. Finally, we analyze the effect of microstructural features of graphite electrodes on transport properties such as the effective diffusivity.
\end{abstract}

\section{INTRODUCTION}

Electrochemical storage is increasingly gaining more attention nowadays. Although it began essentially at the level of consumer electronics, it has grown in recent years and has become a player in the distributed generation or electric vehicles as well as in large-scale applications (e.g. back-up supply at renewable electricity sources). Li-ion batteries are one of the most significant, but not the only, technology currently being used for electrochemical storage. Nevertheless, it presents some sustainability challenges due to market push. In this sense, the demand for this type of batteries has increased dramatically and it will be very difficult to comply with future market expectations [1]. Hence, to fulfill this goal, we need to find smarter storage devices [2].

Batteries also present certain durability concerns, and these are all related to electrochemical, mechanical, and thermal phenomena [3], which threaten key performance indicators such as capacity, charge kinetics, density, or durability. Although this issues are already evident at the so-called system scale, they are 
strongly linked to microstructural features at the component and material levels. These microstructural aspects include, but are not limited to, morphological and physical properties of the electrodes. For instance, in the case of graphite-based electrodes, as it is the case of Lithium-ion battery (LIB) anodes or recent dual-ion graphite batteries (DIGB) electrodes, key features such as the shape of active material (AM) particles, tortuosity, and porosity, play an important role. As a consequence, the development of enhanced electrodes demands a better understanding of these microstructural properties along with the underlying physics present at these scales of observation.

It is therefore crucial to have a better understanding of the degradation mechanisms limiting battery life [4] to define specific improvement actions, such as developing tailor-made geometries, reinforcing strategies or self-healing mechanisms. In fact, advanced computational tools are becoming very useful with regard to the design of a new family of more reliable battery components which are less harmful to the environment. For instance, high-resolution microscopy allows scanning portions of actual components and realize virtual microstructures [5] on which we can perform numerical simulations involving electrochemical, thermal, or mechanical events. This framework is suitable for evaluating the feasibility of new designs or to gain further insight into certain issues of existing ones.

In this work, we propose a lattice-particle approach to study ionic diffusion across graphite electrodes. In our approach, we generate virtual representative volume elements (RVE) of the electrode material based on its composition instead of scanning actual microstructures. Although [7] deals with a similar problem, only active particles were considered throughout the generation process, whereas we not only consider those, but also the rest of constituents, e.g., carbon additives and binder. These particles are

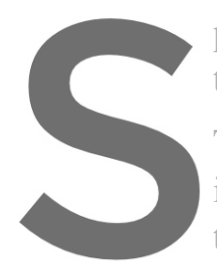
placed within the RVE the RVE also accounts To account for the evolution is solved within a netwo the RVE. We use an expli
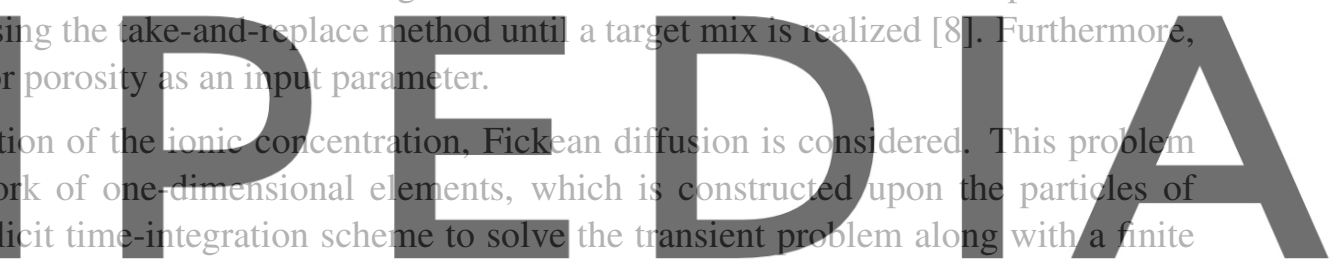

element (FE) discretization of the spatial problem. By using a one-dimensional framework, which is freedom, reducing the computational cost in comparison to the conventional FE-based simulations.

For the transport simulations, we consider Li ions, although our approach can be also applied to other type of species, such as $\mathrm{PF}_{6}^{-}$anions in the case of DIGB with $\mathrm{LiPF}_{6}$ electrolyte, for instance. Finally, we analyze the effect of microstructural features of graphite electrodes on transport properties such as the effective diffusivity.

\section{ELECTRODE MICROSTRUCTURE}

Electrodes are battery components made of different materials, resulting in a complex composite microstructure in which four phases are clearly identified, namely active material, binder, additives, and pores [6]. The active material is where ionic diffusion takes place (i.e., insertion or extraction, also refer to as intercalation or deintercalation, respectively). Typically, Li-ion batteries use graphite as the the active material for Li intercalation in the anode, and lithium-metal oxide, such as NMC (Ni-Mn-Co oxide), in the cathode. In this work, we will assume a spherical shape for the active particles, whose size will depend on the material. In order to enhance electronic transfer and bind active particles together and the 


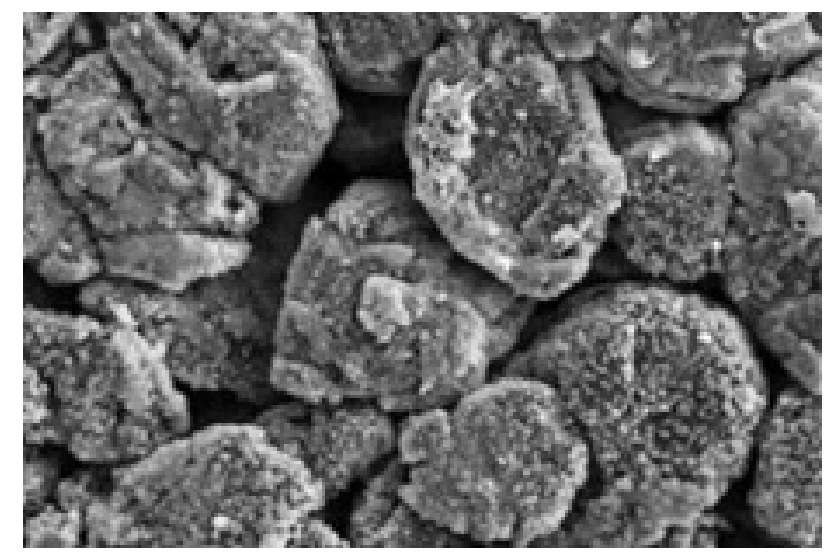

Figure 1: SEM micrograph of graphitic anode exhibiting granluar-like material structure. Reprinted from [9].

mix to the current collectors, the so-called binder is included. Polymeric binders, such as polyvinylidene fluoride (PVDF), are commonly used in the preparation of graphitic slurries, conforming the so-called carbon binder domain (CBD). Also, additives such as carbon black, are included in the electrode synthesis in order to improve the electrode conductivity. These particles can be considered as smaller spheres when compared to those of the active materials. At the microscale, the electrodes resembles a granularlike material structure with porosity ratios that can lie beyond 0.5 , before compaction processes (e.g., calendering) are carried ou

In this work, we consider t on which virtual tests can of these phases are taken intc we consider graphite APs, can be applied to any other
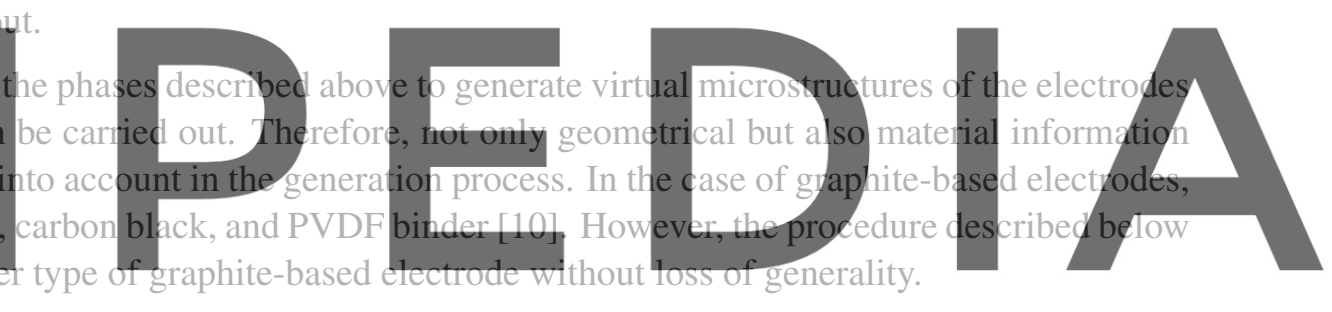

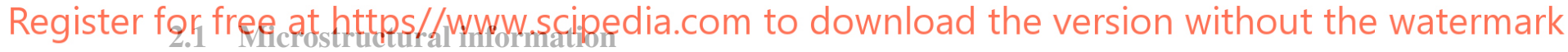

Three dimensional specimens with representative information of the microstructure are generated. The size of this representative volume element (RVE) is an input parameter of this model and must be properly chosen. Several methods can be followed for this purpose $[8,11,12]$ and basically consists in the analysis of the variation of macroscopic properties (e.g., diffusivity) after several realizations of different sizes.

Both geometrical and physical properties of the constituents are accounted in the model as input data:

1. Active material: total volume fraction $\left(v_{a m}\right)$, particle sizes and distribution (in the form of a sieve curve), diffusivity $\left(D_{a m}\right)$.

2. Additive material: total volume fraction $\left(v_{a d}\right)$, particle sizes and distribution, diffusivity $\left(D_{a d}\right)$.

3. Binder: total volume fraction $\left(v_{b i}\right)$, diffusivity $\left(D_{b i}\right)$.

4. Porosity: total volume fraction $\left(v_{p}\right.$ or $\left.\varepsilon\right)$.

The composition of the electrode can be given in terms of the total porosity, $\varepsilon$, and the volume fraction ratios of the constituents (e.g., $v_{a m} / v_{b i} / v_{a d}$ ). 


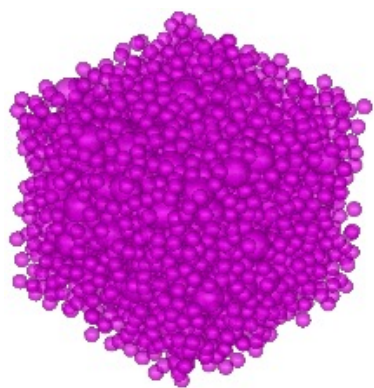

(a)

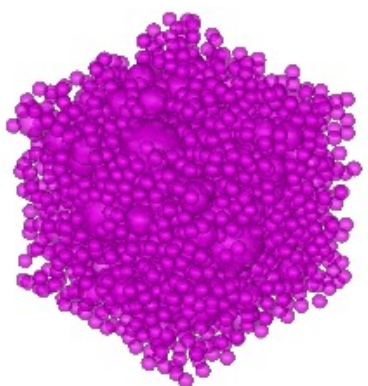

(b)

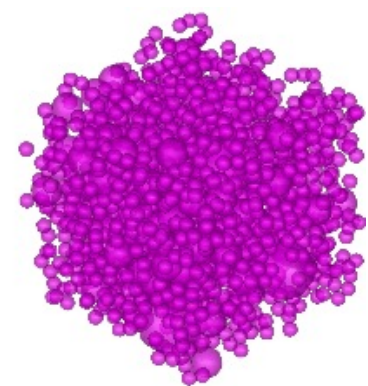

(c)

Figure 2: Virtual realizations of graphite anode microstructures with a) $\varepsilon=0.35$, b) $\varepsilon=0.45$, and c) $\varepsilon=0.55$.

\subsection{Microstructure generation}

Once the yolume fractions of the different constituents have been defined, along with their sizes, we proceed to place them spatially within the cubic domain. As pointed out above, all the particles of both the active material and carbon additives are assumed to be spherical. In the case of the APs, a sieve curve, which is an input parameter, is used to generate the whole distribution of active material particles. Carbon additives particles are modeled with single-size spheres, smaller than that of the smallest AP. All the particles are randomly placed within the RVE following the take-and-place method [8]. The largest particles are placed first following a probability function
following the criterion described in the next section.
relocated at empty positions unti. all the particles a and cathode,

Figure 2 presents three different realizations of
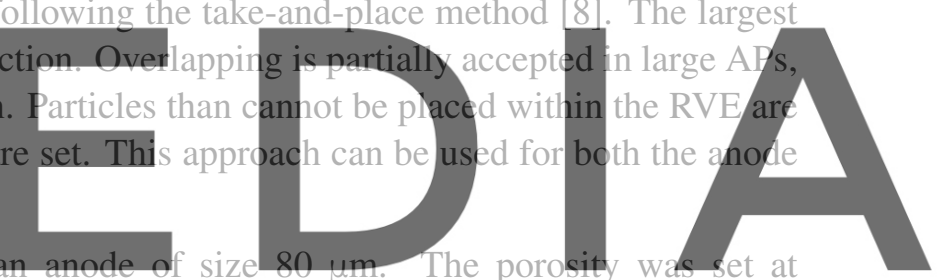

$\varepsilon=0.35,0.45$, and 0.55 . APs of 5,10 , and $20 \mu \mathrm{m}$ with fraction volumes of $0.5,0.3$, and 0.2 are con-

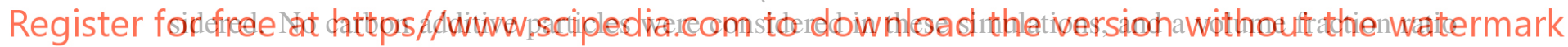
$v_{a m} / v_{b i}=0.95 / 0.05$ was defined. Figure 3 presents three realizations for a cathode of the same size and similar porosity values. In this case, additive particles (depicted in cyan) of $4 \mu \mathrm{m}$ was chosen and a volume fraction ratio of $v_{a m} / v_{b i} / v_{a d}=0.95 / 0.05 / 0.05$ was defined.

\section{MATHEMATICAL MODEL OF ION DIFFUSION IN ACTIVE MATERIALS}

Let us suppose that the ion diffusion process occurs within a three dimensional cube of size $d,[0, d]^{3} \subset \mathbb{R}^{3}$ having a number of $N$ particles. The faces of the cube whose unit normal vector is in the $x$ direction will be denoted by $\pi_{Z=0}$ for the face of the cube in the $Z=0$ plane and $\pi_{Z=d}$ for the face of the cube in the plane $Z=d$.

We will use the symbols $\Omega_{i}$ and $\Gamma_{i}$ to denote the inner volume and the surface boundary of the $i$-th particle. Sometimes, we will use the notation $\bar{\Omega}_{i}:=\Omega_{i} \cup \Gamma_{i}$ to refer to both, the volumen and boundary 


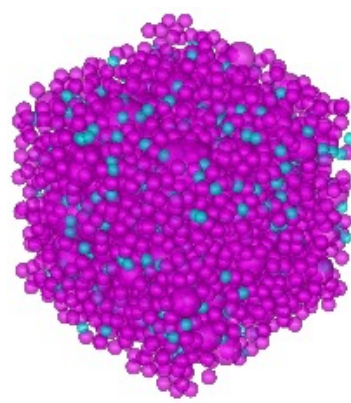

(a)

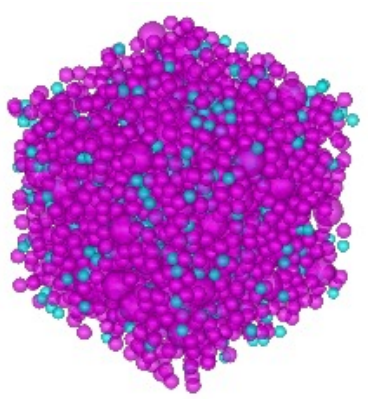

(b)

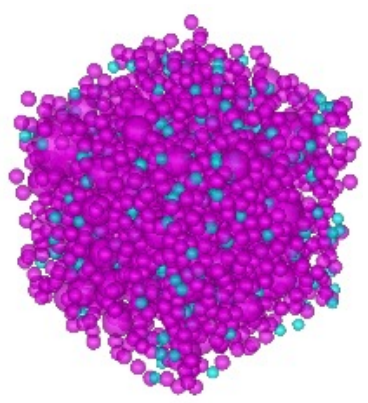

(c)

Figure 3: Virtual realizations of graphite cathode microstructures with a) $\varepsilon=0.35$, b) $\varepsilon=0.45$, and c) $\varepsilon=0.55$. Magenta particles correspond to APs and cyan particles to additive particles.

of the i-th particie, i.e. $\bar{\Omega}_{i}:=\Omega_{i} \cup \Gamma_{i}$. Let us also call

$$
U:=\bigcup_{i=1}^{N} \bar{\Omega}_{i},
$$

the space of the cube occupied by the particles.

In order to analyze the

equation, a parabolic p

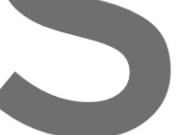

\section{where $c=c(\mathbf{x}, t)$ is the $\mathbf{L}$}
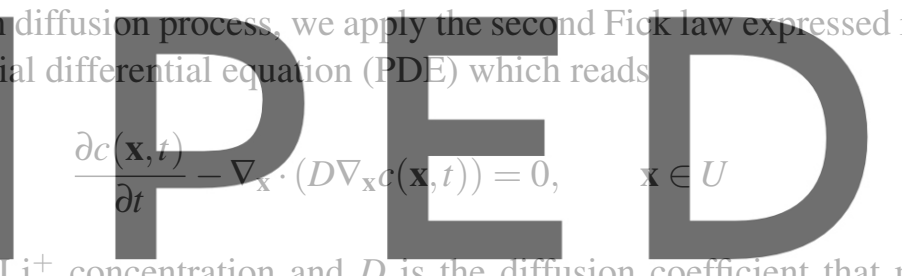

Register for free at https//wwW.scipedia.com to download the version without the watermark

There are two boundary value problems based on (1) of our interest: the transient problem and the steady

state problem. On the one hand, the transient problem provides the evolution of the concentration with

time whilst the steady state problem is useful to determine the average diffusivity of the electrode.

\subsection{Transient problem}

For the transient problem, we shall use the Neumann boundary conditions given in terms of the conormal derivative of the concentration, i.e., the species flux given by the following formula

$$
\boldsymbol{J}(\mathbf{x}, t) \cdot \mathbf{n}:=-D \frac{\partial c}{\partial \mathbf{n}}=-D \nabla_{\mathbf{x}} c(\mathbf{x}, t) \cdot \mathbf{n}
$$

Hence, the Neumann boundary condition for the transient problem is

$$
\boldsymbol{J}(\mathbf{x}, t) \cdot \mathbf{n}:= \begin{cases}\mathbf{J}_{0} & \boldsymbol{x} \in \Gamma_{i} \text { and } \Gamma_{i} \cap \pi_{Z=0} \neq \emptyset \\ 0 & \text { else }\end{cases}
$$


To obtain a uniquely solvable boundary value problem using (1) and (2), we also need the initial condition

$$
c(\mathbf{x}, 0)=c_{0}, \quad \forall \mathbf{x} \in U .
$$

Consequently, equations (1)-(3) give an interior Neumann boundary value problem to be solved in a three dimensional domain with smooth boundary. This problem is well-posed[14] and has as solution $c(\mathbf{x}, t)$ for all $\mathbf{x} \in U$ and $t>0$.

\subsection{Dirichlet steady-state problem}

The steady-state problem for the PDE given in (1) with Dirichlet boundary conditions can be used to estimate the average diffusivity of the electrode.

Let us note that the steady state equation for (1) is obtained as a result of removing the time-derivative terms

$$
-\nabla_{\mathbf{X}} \cdot\left(D \nabla_{\mathbf{x}} c(\mathbf{x}, t)\right)=0, \quad \mathbf{x} \in U
$$

By preseribing a two different concentrations $c_{0}$ and $c_{1}$ at two opposite surfaces, e.g. $\pi_{Z=0}$ and $\pi_{Z=d}$, we can determine the average diffusivity of the electrode $\left(\left\langle D_{x}\right\rangle\right)$ in the direction of $\mathbf{x}$ by measuring the corresponding reaction fluxes $\left(J_{S}\right)$ through these two surfaces and using the formula

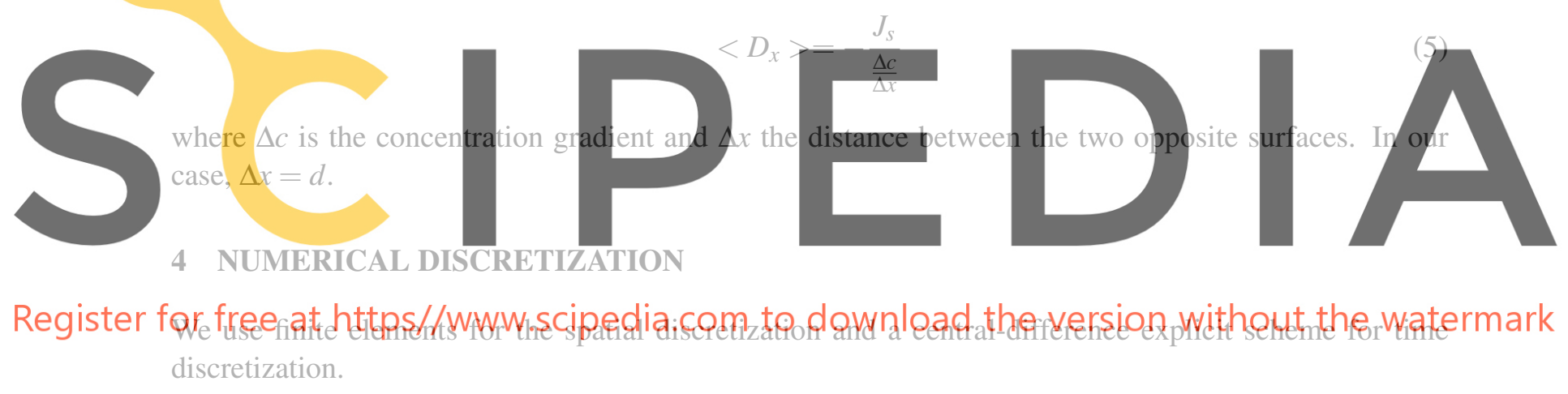

\subsection{Time discretization}

The finite difference scheme applied to time-discretize is based on the evaluation of the concentration rate vector at an instant $t=t_{n}$ using the formula

$$
\dot{\mathbf{c}}_{n}=\frac{\mathbf{c}_{n+1}-\mathbf{c}_{n-1}}{2 \Delta t}
$$

where $\mathbf{c}_{n+1}$ and $\mathbf{c}_{n-1}$ are the concentration vectors at instants $t_{n+1}$ and $t_{n-1}$, respectively. $\Delta t$ is the time step size, e.g., $\Delta t=t_{n+1}-t_{n}$.

Thus, the concentration at time step $t_{n+1}$ is approximated as $\mathbf{c}_{n+1}=\mathbf{c}_{n-1}+2 \Delta t \dot{\mathbf{c}}_{n}$. Let us remark that forward Euler scheme is used for the first iteration $(n=1)$, thus, $\mathbf{c}_{1}=\mathbf{c}_{0}+\Delta t \dot{\mathbf{c}}_{0}$, with $\dot{\mathbf{c}}_{0}=\mathbf{M}_{d}^{-1}\left[\mathbf{q}_{0}-\mathbf{K}_{\mathrm{d}} \mathbf{c}_{0}\right]$. 


\subsection{Space discretization}

It is well known that the explicit discretization of time along with the space discretization using the finite element method to (1) leads to the following system of linear equations [15]

$$
\mathbf{M}_{\mathrm{d}} \dot{\mathbf{c}}+\mathbf{K}_{\mathrm{d}} \mathbf{c}=\mathbf{q}
$$

where $\mathbf{M}_{\mathrm{d}}$ is the so-called capacity matrix, $\mathbf{K}_{\mathrm{d}}$ is the diffusion matrix, $\mathbf{c}$ is the concentration vector, $\dot{\mathbf{c}}$ is the concentration rate vector, and $\mathbf{q}$ is the flux vector.

\subsection{Lattice-particle formulation}

The spatial discretization of the domain $\Omega$ is based on the lattice-particle approach [16]. The domain is discretized into a network of one-dimensional flow elements connecting the centroids of the particles of the microstructure. Each 1D flow element has its own capacity and diffusion matrix, that depends on the relative distances of the particles. The particles interaction is established a priori using Delaunay's triangulation [8], resulting in a 3D mesh. An internal parameter $\beta$ is used to control overlapping between large particles. The binder is modeled as a meniscus formed between particles up to a certain distance [6], and its behavior is lumped into the element matrices.

For any element of the mesh, the diffusion element matrix reads as:
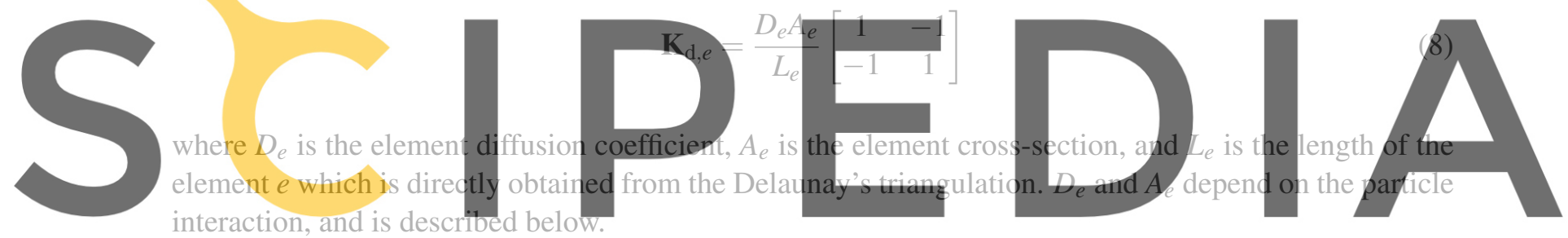
interaction, and is described below.

Register for free at hand the capacity element matrix reads as:

$$
\mathbf{M}_{\mathrm{d}, e}=\frac{A_{e} L_{e}}{\omega}\left[\begin{array}{ll}
2 & 1 \\
1 & 2
\end{array}\right]
$$

being $\omega$ a dimensional factor used in this type of network models. In the case of 3D lattices, $\omega=3$ [17].

\section{Definition of element properties}

The definition of the element properties $D_{e}$ and $A_{e}$ depends on the distance between two interacting particles $i$ and $j, L_{e}$, and their respective diameters, $d_{i}$ and $d_{j}$. We define the meniscus length, $l_{m}$, as the gap existing between two interacting particles, $l_{m}=L_{e}-d_{i} / 2-d_{j} / 2$. Thus, depending on the value of $l_{m}$, three cases can be identified:

(i) $l_{m}<0$. In this case, particles overlap (Figure 4a). This is only possible for large particles (i.e., active material particle). Therefore, the element diffusivity is that of the active material, $D_{e}=D_{a m}$. The cross-section of the element is defined in terms of the intersection radius, $h$. Thus, $A_{e}=\pi h^{2}$. 


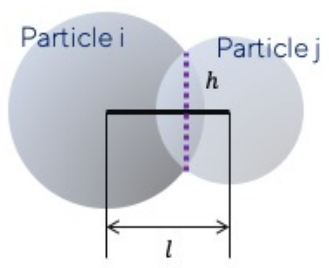

(a)

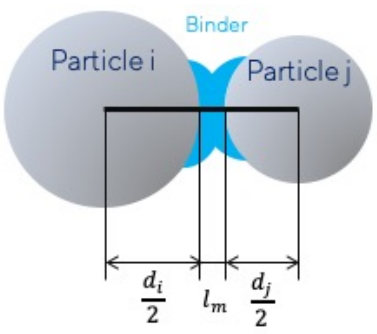

(b)

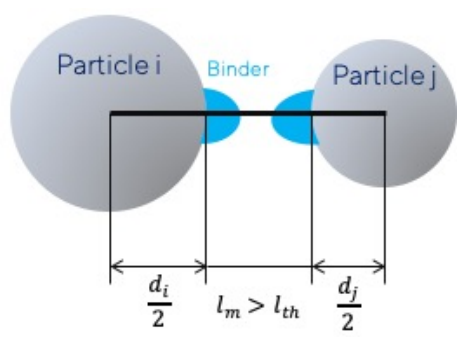

(c)

Figure 4: Possible particle interactions: a) overlapping $\left(l_{m} \leq 0\right)$, b) binding $0 \leq l_{m} \leq l_{t h}$, and c) no interaction $l_{m}>l_{t h}$.

(ii) $0 \leq l_{m} \leq l_{t h}$, where $l_{t h}$ is an interaction threshold until which particles are binded (Figure $4 \mathrm{~b}$ ). In this case, two phases are present: active material and binder. We assume a serial coupling (resistance analogy) of the phases interacting:

$$
\left(\frac{D_{e} A_{e}}{L_{e}}\right)^{-1}=\left(\frac{2 D_{a m} A_{e}}{d_{i}}\right)^{-1}+\left(\frac{D_{b i} A_{m}}{l_{m}}\right)^{-1}+\left(\frac{2 D_{a m} A_{e}}{d_{j}}\right)^{-1}
$$

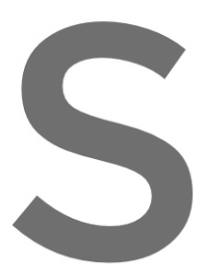

where $D_{b i}$ is the diffusivity of the binder. The area of the meniscus, $A_{m}$, is obtained from volume
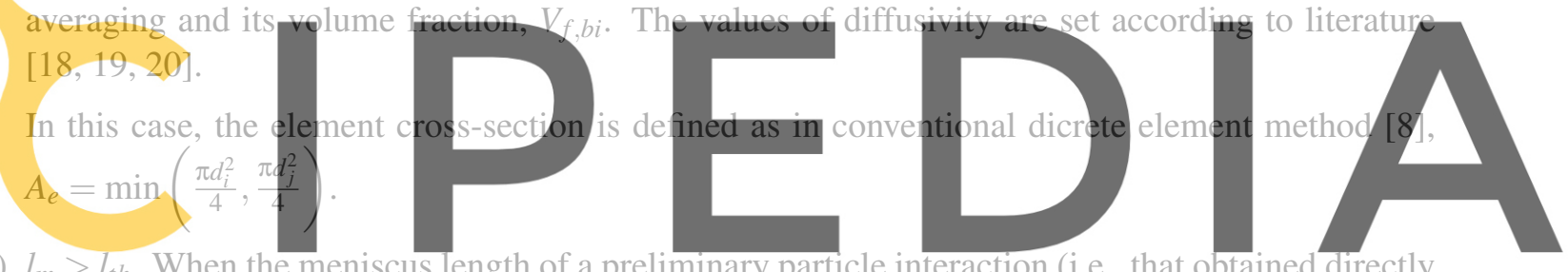

(iii) $l_{m}>l_{t h}$. When the meniscus length of a preliminary particle interaction (i.e., that obtained directly

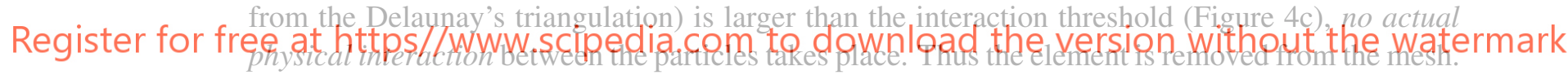

\section{IMPLEMENTATION}

\subsection{Transient problem}

Making use of equations 8 and 9, we can assembly the global capacity and diffusion matrices, $\mathbf{M}_{\mathrm{d}}$ and $\mathbf{K}_{\mathrm{d}}$, respectively, which are required to solve equation 7 within the FE framework. Moreover, we approximate the concentration rate at instant $t_{n}$ as:

$$
\dot{\mathbf{c}}_{n}=\mathbf{M}_{\mathrm{d}}^{-1}\left[\mathbf{q}_{n}-\mathbf{K}_{\mathrm{d}} \mathbf{c}_{n}\right]
$$

The nodes of the mesh correspond to the centroids of the particles. At each node we define one degree of freedom, i.e., average concentration of the particle, $c$. Therefore, the number of degrees of freedom of the system equals the total number of particles. 


\subsection{Steady-state problem}

The steady-state problem can be also solved in order to evaluate the macroscopic diffusion coefficient of the electrode.

To solve the steady-state problem, $\mathbf{K}_{d} \mathbf{c}=\mathbf{q}$, we chose the conjugate gradient (CG) method with Jacobian preconditioning to improve the conditioning number of the matrix. The CG method is one of the most used iterative solvers in sparse systems of linear equations, as is the case of our study. This method was already used by other authors for lattice modeling [21]. This implicit approach solves the system iteratively, but when certain conditions are satisfied, it may become a fast approach, especially in the case of evolving systems (which is the scope of our future research). For an updated overview of this method and its application to large-scale systems, refer to [22].

\section{EFFECT OF MICROSTRUCTURE ON ELECTRODE PERFORMANCE}

In this section we present preliminary results of our model on two different applications. In the first case, we evaluate the effect of porosity in the macroscopic diffusion coefficient of the electrode using steady-state simulations, while in the second case we present the time-dependent response of virtual microstructures under charge and discharge conditions.

For the first case, we consider the boundary conditions as described in section 5.2 and measure the effective diffusivity of the electrode (Figure 5). Four different values of porosity are accounted for, namely $\varepsilon=$

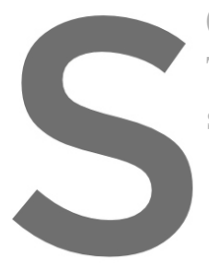
$0.3,0.4,0.5$, and 0.6 . For the sir
This results into different RVE
shown in Table 6 and are in agree
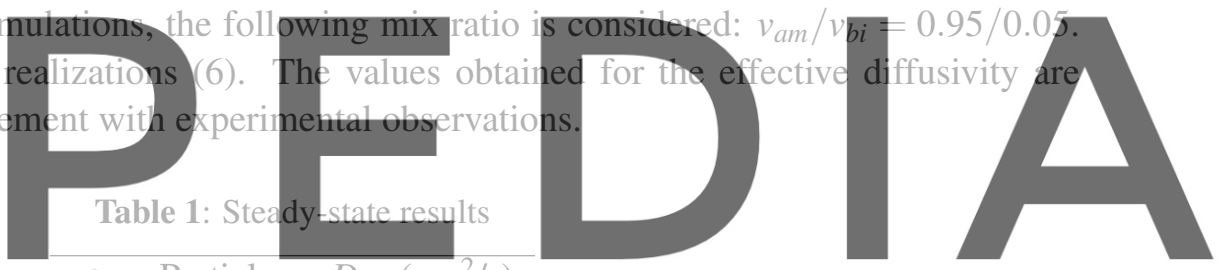

$\varepsilon \quad$ Particles $D_{\text {eff }}\left(\mu \mathrm{m}^{2} / \mathrm{s}\right)$

Register for free at https//www.scipedia.com to downifoad the version without the watermark

\begin{tabular}{lll}
\hline 0.5 & 2011 & 0.0095 \\
\hline 0.6 & 1606 & 0.0084
\end{tabular}

The second application is the simulation of electrode discharge. For this reason, the electrode is initially charged with a Li-ion concrentration of $30000 \mathrm{~mol} / \mathrm{m}^{3}$. In this example we show the discharging process of the electrode subject to at $0.5 \mathrm{C}$-rate galvonstatic conditions at one boundary. In Figure 7 we see the evolution of the Li-ion concentration along the discharge process.

\section{CONCLUSIONS}

In this work, we have presented a novel lattice-particle model to analyze Li-ion diffusion, which is a key physical mechanism to understand important performance features of a battery (e.g. charging/ discharging times and capacity fade). Most of the existing works in the literature solve this problem using continuum finite elements. However, when it comes to microstructural modeling, high spatial and temporal resolutions are required. Therefore, alternative simulation techniques that alleviate such 


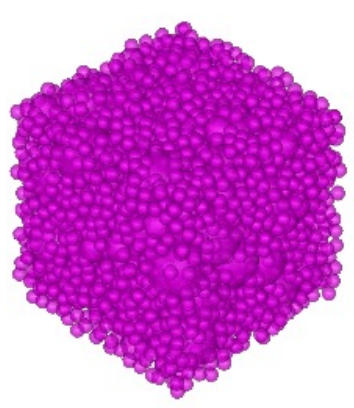

(a)

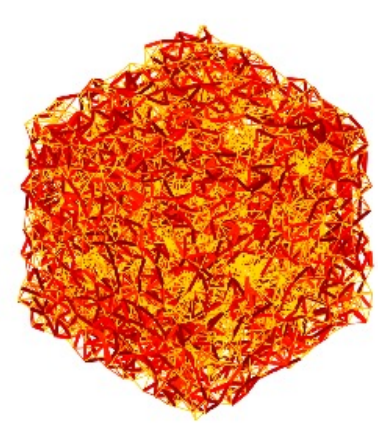

(b)
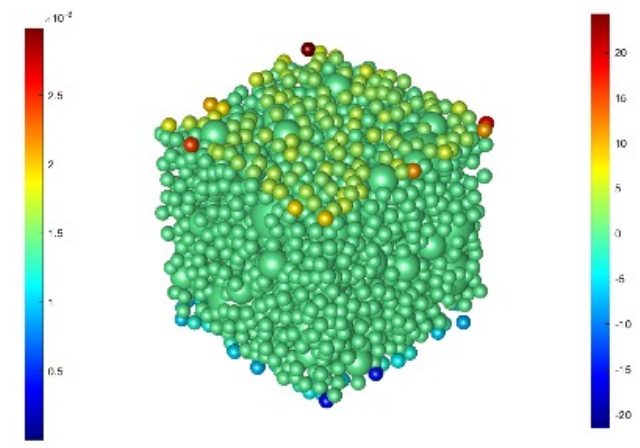

(c)

Figure 5: Steady-state simulation of an anode: a) microstructure, b) mesh, and c) reaction fluxes.
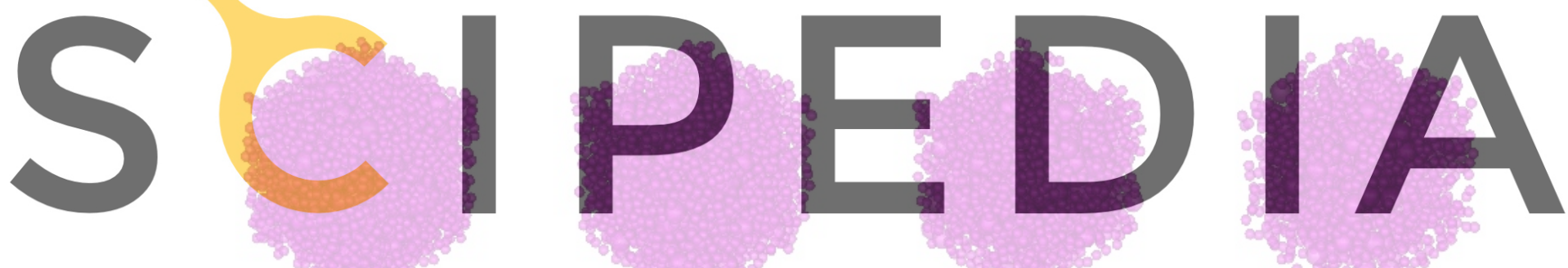

Register for free at https//www.scipedia.com to download the version without the watermark

(a)

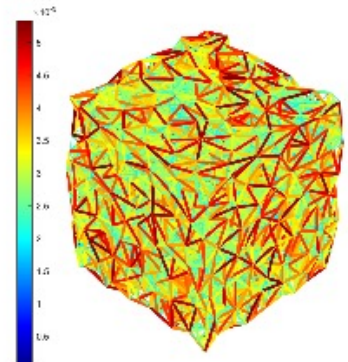

(e) (b)

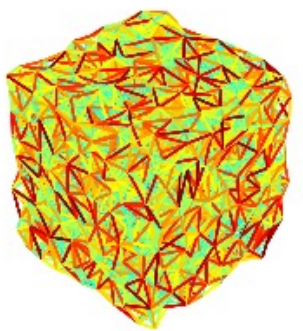

(f) (c)

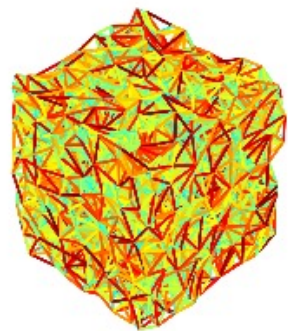

(g) (d)

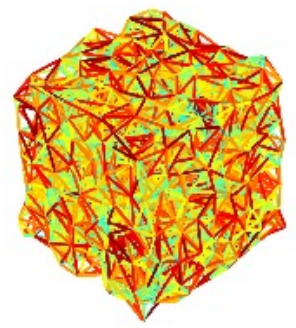

(h)

Figure 6: RVE realizations and resulting mesh: a-e) $\varepsilon=0.3$, b-f) $\varepsilon=0.4$, c-g) $\varepsilon=0.5$, d-h) $\varepsilon=0.6$. 


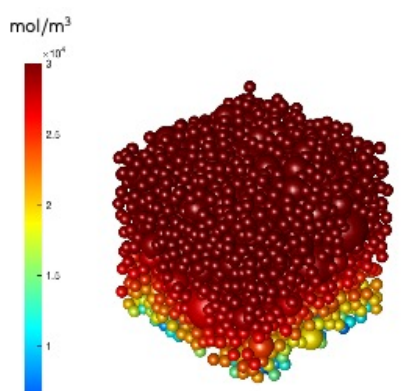

(a)

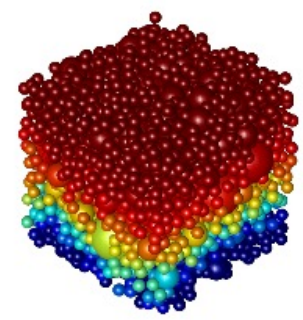

(b)

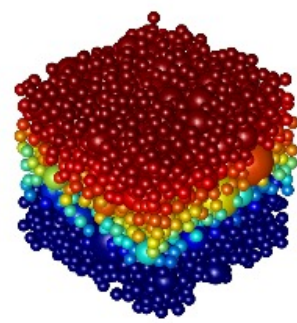

(c)

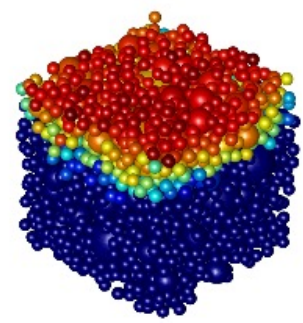

(d)

Figure 7: Galvanostatic discharge (at 0.5 C-rate) of the anode: a) $500 \mathrm{~s}$, b) $2000 \mathrm{~s}, \mathrm{c}) 3500 \mathrm{~s}$, and d) $7500 \mathrm{~s}$.

computational demands are of great help, especially in materials design for electrodes.

As shown herein, our approach dramatically reduces the number of degrees of freedom while keeping relevant microstructural features at the very local scale. We have used this method to study the effect of porosity and local diffusion properties (i.e., that of active materials) in the effective diffusivity of the electrode, which is an important parameter regarding the macroscopic behavior of the cell.This is can be measured by means of steady-state simulations. However, we also present transient simulations of virtual electrode microstructures of charging/discharging processes.

Regarding the numerical implementation of solving schemes, we used the Conjugate Gradient method with Jacobian preconditioning to solve the steady-state problem and the central-difference time integration scheme to solve the transient problem.

Finally, we have focused on Li-ion diffusion in graphite electrodes. However, we plan to implement the mechanical problem [16] in order to account for diffusion-induced damage. This will allow us to characterize the cycling behavior and hysteresis of the electrodes. This approach can be used for both positive and negative electrodes and in future work we will replace $\mathrm{Li}^{+}$by $\mathrm{PF}_{6}^{-}$as anion.

\section{ACKNOWLEDGMENTS}

This work was partially funded by the Consejería de Economía, Conocimiento, Empresas y Universidad (Junta de Andalucía), under the project grant PY18-RE-0023, "Multiscale, multiphysics simulation platform for virtual design of batteries: The VirtualBats Project".

\section{REFERENCES}

[1] T. Tsujikawa, K. Yabuta, M. Arakawa, and K. Hayashi. Safety of large-capacity lithium-ion battery and evaluation of battery system for telecommunications. Journal of Power Sources, 244:11-16, 2013 .

[2] R. Wagner, N. Preschitschek, S. Passerini, J. Leker, and M. Winter. Current research trends and prospects among the various materials and designs used in lithium-based batteries. Journal of Applied Electrochemistry, 43(5):481-496, 2013. 
[3] P. Arorat, R. E. White, and M. Doyle Capacity Fade Mechanisms and Side Reactions in Lithium-Ion Batteries. Journal of the Electrochemical Society, 145(10):3467-3667, 1998.

[4] J.M. Tarascon and M. Armand Issues and challenges facing rechargeable lithium batteries. Nature, 414:359-367, 2001.

[5] X. Lu, A. Bertei, D.P. Finegan, C. Tan, S.R. Daemi, J.S. Weaving, K.B. O'Regan, T. M. M. Heenan, G. Hinds, E. Kendrick, D. J. L. Brett, and P.R. Shearing. 3D microstructure design of lithium-ion battery electrodes assisted by X-ray nano-computed tomography and modelling. Nature Communications, 11(1):2079, 2020.

[6] C.-F. Chen, A. Verma, and P.P. Mukherjee. Probing the Role of Electrode Microstructure in the Lithium-Ion Battery Thermal Behavior. Journal of The Electrochemical Society, 164(11):E3146E3158, 2017.

[7] D. Westhoff, I. Manke, and V. Schmidt. Generation of virtual lithium-ion battery electrode microstructures based on spatial stochastic modeling. Computational Materials Science, 151:53-64, 2018 .

[8] F. Montero-Chacón and F. Medina. A lattice-particle approach to determine the RVE size for quasibrittle materials. Engineering Computations, 30(2):246-262, 2013.

[9] Y. Dong, J. Demeaux, Y. Zhang, M. Xu, L. Zhou, A.D. MacIntosh, and B.L. Lucht. Improving the Performance at Elevated Temperature of High Voltage Graphite/LiNi 0.5 Mn 1.5 O 4 Cells with Added Lithium Catechol Dimethyl Borate. Journal of The Electrochemical Society, 164(2):A128A136, 2017.

[10] B. Dyatkin, J. Halim, and J. Read. Electrode Surface Composition of Dual-Intercalation, AllGraphite Batteries. C, 3(4):5, 2017.

[11] I.M. Gitman, H. Askes, and L.J. Sluys. Representative volume: Existence and size determination. Engineering Fracture Mechanics, 74(16):2518-2534, nov 2007.

[12] M. Zhang, G. Ye, and K. van Breugel. Multiscale lattice Boltzmann-finite element modelling of chloride diffusivity in cementitious materials. Part I: Algorithms and implementation. Mechanics Research Communications, 58:53-63, 2014.

[13] X. Zhang, W. Shyy, and A.M. Sastry. Numerical Simulation of Intercalation-Induced Stress in Li-Ion Battery Electrode Particles. Journal of The Electrochemical Society, 154(10):A910, 2007.

[14] J.R. Cannon The one-dimensional heat equation. Cambridge University Press, 1984.

[15] C. Johnson Numerical solution of partial differential equations by the finite element method. Courier Corporation, 2012.

[16] F. Montero-Chacón, H. Cifuentes, and F. Medina. Mesoscale Characterization of Fracture Properties of Steel Fiber-Reinforced Concrete Using a Lattice-Particle Model. Materials, 10(2):207, 2017.

[17] J.E. Bolander and S. Berton. Simulation of shrinkage induced cracking in cement composite overlays. Cement and Concrete Composites, 26(7):861-871, 2004.

[18] P. Yu, B. N. Popov, J. A. Ritter, and R. E. White. Determination of the Lithium Ion Diffusion 
Coefficient in Graphite. Journal of The Electrochemical Society, 146(1):8-14, 1999.

[19] Y. Zhu and C. Wang. Galvanostatic Intermittent Titration Technique for Phase-Transformation Electrodes. The Journal of Physical Chemistry C, 114(6):2830-2841, 2010.

[20] M. Chouchane, A. Rucci, T. Lombardo, A. C. Ngandjong, and A.A. Franco. Lithium ion battery electrodes predicted from manufacturing simulations: Assessing the impact of the carbon-binder spatial location on the electrochemical performance. Journal of Power Sources, 444:227285, 2019.

[21] E. Schlangen and E.J. Garboczi. Fracture simulations of concrete using lattice models: Computational aspects. Engineering Fracture Mechanics, 57(2-3):319-332, 1997.

[22] J. Bolz, I. Farmer, E. Grinspun, and P. Schröder. Sparse matrix solvers on the GPU. ACM Transactions on Graphics, 22(3):917-924, 2003. 